\title{
Nano-XANES: Chemical Mapping with Hard X-rays
}

\author{
Ajith Pattammattel, ${ }^{1}$ Ryan Tappero, ${ }^{1}$ Mingyuan \\ Ge, ${ }^{1}$ Xiaojing Huang, ${ }^{1}$ Yong Chu, ${ }^{1}$ Yuan Gao ${ }^{1}$ \\ and Hanfei Yan ${ }^{1}$ \\ ${ }^{1}$ National Synchrotron Light Source II, \\ Brookhaven National Laboratory, Upton, NY, \\ USA
}

X-ray absorption spectroscopy (XAS) is one of the widely used technique across multiple disciplines to probe chemical and valence state of an atom. XAS is a useful tool for speciation of heterogenous biogeochemical and environmental samples due to the element specificity and high penetration depth of hard X-rays $(>5 \mathrm{keV})$. Recently the transport, transformation and fate of nano-scale materials in the environment have paid larger attention due to their health and environmental impacts. However, most XAS characterization tools lack spatial resolution to probe these nano systems. At the 'state-of-the-art' hard X-ray nanoprobe (HXN) beamline at NSLS-II, scanning hard $\mathrm{x}$-ray imaging as low as $10 \mathrm{~nm}$ spatial resolution was well established. We extend the capability at HXN to nanoscale chemical imaging with X-ray absorption Fine Structure (XANES). In this presentation we will discuss recent developments, advantages, limitations and sample considerations of nanoXANES. Fe(0) and $\mathrm{Fe}(\mathrm{III})$ nanoparticle mixture was used as a model system for technique development and establishing the data processing. After the successful demonstration of $\mathrm{Fe}(0)$ and $\mathrm{Fe}(\mathrm{III})$ speciation in the $2 \mathrm{D}$ matrix, we applied the technique to a challenging system of iron phosphide nanoparticles in micron-size lithium iron phosphate. Due to their chemical similarity, size differences and low abundance $(<5 \%)$ of the Fe-phosphide phases, traditional several microscopic and spectroscopic techniques was insufficient for speciation. We showed that nano-XANES bundled with spatial resolution, high detection sensitivity and penetration power can successfully resolved both phases with clear grain boundaries. To our best knowledge, hard X-ray fluorescent yield nano-XANES that can resolve chemical state variations with nanoscale spatial resolution is not achieved previously. Current development can address several grand challenges in nanoscale speciation of materials relevant to broad areas in biogeochemistry and environmental sciences. 
This abstract is too long to be accepted for publication.

Please revise it so that it fits into the column on one page. 